\title{
Silencing of miR-195 reduces diabetic cardiomyopathy in C57BL/6 mice
}

\author{
Dong Zheng ${ }^{1,2,3,4} \cdot$ Jian $\mathrm{Ma}^{2,3,4} \cdot$ Yong $\mathrm{Yu}^{5} \cdot$ Minghui $\mathrm{Li}^{5} \cdot \mathrm{Rui} \mathrm{Ni}^{2,3,4} \cdot$ Grace Wang $^{4}$.

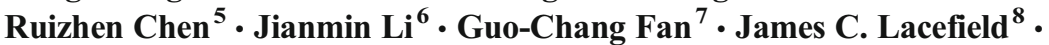 \\ Tianqing Peng ${ }^{1,2,3,4}$
}

Received: 5 November 2014 / Accepted: 14 April 2015 / Published online: 21 May 2015

(C) Springer-Verlag Berlin Heidelberg 2015

\begin{abstract}
Aims/hypothesis MicroRNAs (miRs) have been suggested as potential therapeutic targets for heart diseases. Inhibition of miR-195 prevents apoptosis in cardiomyocytes stimulated with palmitate and transgenic overexpression of miR-195 induces cardiac hypertrophy and heart failure. We investigated whether silencing of miR-195 reduces diabetic cardiomyopathy in a mouse model of streptozotocin (STZ)-induced type 1 diabetes.

Methods Type 1 diabetes was induced in C57BL/6 mice (male, 2 months old) by injections of STZ.

Results MiR-195 expression was increased and levels of its target proteins (B cell leukaemia/lymphoma 2 and sirtuin 1) were decreased in STZ-induced type 1 and $d b / d b$ type 2 diabetic mouse hearts. Systemically delivering an anti-miR-195 construct knocked down miR-195 expression in the heart, reduced caspase-3 activity, decreased oxidative stress, attenuated myocardial hypertrophy and improved myocardial function in STZ-induced mice with a concurrent upregulation of B
\end{abstract}

Dong Zheng, Jian Ma and Yong Yu contributed equally to this work.

Electronic supplementary material The online version of this article (doi:10.1007/s00125-015-3622-8) contains peer-reviewed but unedited supplementary material, which is available to authorised users.

Tianqing Peng

tqpeng@suda.edu.cn

1 Institutes of Biology and Medical Sciences, Soochow University, Suzhou, Jiangsu Province, China 215123

2 Critical Illness Research, Lawson Health Research Institute, VRL 6th Floor, A6-140, 800 Commissioners Road, London, ON, Canada N6A 4G5

3 Department of Medicine, The University of Western Ontario, London, ON, Canada cell leukaemia/lymphoma 2 and sirtuin 1. Diabetes reduced myocardial capillary density and decreased maximal coronary blood flow in mice. Knockdown of miR-195 increased myocardial capillary density and improved maximal coronary blood flow in diabetic mice. Upregulation of miR-195 sufficiently induced apoptosis in cardiomyocytes and attenuated the angiogenesis of cardiac endothelial cells in vitro. Furthermore, inhibition of miR-195 prevented apoptosis in cardiac endothelial cells in response to NEFA, an important feature of diabetes.

Conclusions/interpretation Therapeutic silencing of miR-195 reduces myocardial hypertrophy and improves coronary blood flow and myocardial function in diabetes, at least in part by reducing oxidative damage, inhibiting apoptosis and promoting angiogenesis. Thus, miR-195 may represent an alternative therapeutic target for diabetic heart diseases.

Keywords Angiogenesis · Apoptosis - Cardiomyopathy · Diabetes $\cdot$ Hypertrophy $\cdot$ miR-195 $\cdot$ Myocardial dysfunction

4 Department of Pathology, The University of Western Ontario, London, ON, Canada

5 Zhongshan Hospital of Fudan University, Shanghai, China

6 Department of Pathology, The First Affiliated Hospital of Wenzhou Medical College, Wenzhou, Zhejiang, China

7 Department of Pharmacology and Cell Biophysics, University of Cincinnati College of Medicine, Cincinnati, OH, USA

8 Electrical and Computer Engineering, Medical Biophysics, Robarts Research Institute, University of Western Ontario, London, ON, Canada 


$\begin{array}{ll}\text { Abbreviations } & \\ \text { Ad-miR-195 } & \text { Adenoviral vectors containing miR-195 } \\ \text { Ad-gal } & \text { Adenoviral vectors containing } \beta \text {-gal } \\ \text { ALT } & \text { Alanine transaminase } \\ \text { AST } & \text { Aspartate transaminase } \\ \text { BCL-2 } & \text { B cell leukaemia/lymphoma 2 } \\ \text { DCF-DA } & \text { 2,7-Dichlorodihydro-fluorescein diacetate } \\ \text { FS } & \text { Fractional shortening } \\ \text { LV } & \text { Left ventricular } \\ \text { miR } & \text { MicroRNA } \\ \text { MOI } & \text { Multiplicity of infection } \\ \text { ROS } & \text { Reactive oxygen species } \\ \text { Sirt1 } & \text { Sirtuin 1 } \\ \text { STZ } & \text { Streptozotocin }\end{array}$

\section{Introduction}

Globally, the number of adults affected by diabetes is rapidly growing and is estimated to reach nearly 400 million by 2030 [1]. Diabetes induces microvascular and macrovascular dysfunction [2] and cardiac structure and function can be affected directly, a condition called diabetic cardiomyopathy, independent of coronary artery disease and hypertension [3]. Diabetic cardiomyopathy has a long latent phase during which it is completely asymptomatic. An early sign of the disease is mild left ventricular (LV) diastolic dysfunction with preserved systolic function. Diabetic cardiomyopathy is characterised functionally by cardiac hypertrophy, loss of cardiomyocytes, interstitial fibrosis and diastolic dysfunction followed by systolic dysfunction [3]. However, the pathophysiology of diabetic cardiomyopathy is incompletely understood and currently no specific therapy is available.

MicroRNAs (miRs) are a class of short RNA molecules, on average 22 nucleotides long, encoded within the genome and derived from endogenous small hairpin precursors. miRs negatively regulate gene expression by targeting the $3^{\prime}$ untranslational region of specific mRNA for transcript degradation or translational repression [4]. MiRs are involved in a wide range of pathophysiological cellular processes including development, differentiation, growth, metabolism, survival/ death and tumour formation [5-10]. Aberrant expression of miRs has been linked to a number of myocardial pathological conditions including hypertrophy, fibrosis, apoptosis, regeneration, arrhythmia and heart failure [10-16]. As such, miRs have been suggested as novel therapeutic targets for heart diseases $[17,18]$. Previous studies have demonstrated that miR-195 is associated with cardiac hypertrophy and heart failure $[12,19,20]$. Forced overexpression of miR-195 is sufficient to induce cardiac hypertrophy and heart failure in transgenic mice [19]. We have recently demonstrated that miR-195 promotes NEFA-induced apoptosis in cardiomyocytes by downregulating B cell leukaemia/lymphoma 2 (BCL-2) and sirtuin 1 (Sirt1) [21] This suggests that miR-195 has a potential role in diabetic cardiomyopathy since NEFA increase in diabetes. However, it is not known whether therapeutic inhibition of miR-195 reduces diabetic cardiomyopathy.

The present study examined the role of miR-195 in diabetic cardiomyopathy and investigated whether therapeutic inhibition of miR-195 reduces diabetic cardiomyopathy in a mouse model of streptozotocin (STZ)-induced type 1 diabetes.

\section{Methods}

Animals The principles of laboratory animal care (NIH Publication No. 85-23, revised 1985) were followed, as well as specific national laws where applicable. All experimental procedures were approved by the Animal Use Subcommittee at the University of Western Ontario, Canada. C57BL/6 mice, $d b / \pm$ and $d b / d b$ mice were purchased from the Jackson Laboratory (Sacramento, CA, USA). Adult male rats (SpragueDawley, 150-200 g body weight) were purchased from Charles River Laboratories (Montreal, QC, Canada).

Experimental protocol Diabetes was induced in mice (male, 2 months old) by consecutive peritoneal injections of STZ (50 mg kg ${ }^{-1}$ day $^{-1}$; Sigma, Toronto, ON, Canada) for 5 days. The mice were considered diabetic and used for the study if they had hyperglycaemia $(\geq 15 \mathrm{mmol} / \mathrm{l})$ at $72 \mathrm{~h}$ after STZ injection. Mice treated with citrate buffer were used as nondiabetic controls (blood glucose $<12 \mathrm{mmol} / \mathrm{l}$ ).

To silence miR-195 expression in hearts, we used antimiR-195 miR construct (miRZip-195; System Biosciences, Mountain View, CA, USA). A construct containing the scramble hairpin (miRZip00) served as a control. MiRZip-195 or miRZip00 $(60 \mu \mathrm{g})$ was mixed with $40 \mu \mathrm{l}$ of nanoparticlebased transfection reagent (Altogen Biosystem, Las Vegas, NV, USA) with a total volume of $500 \mu \mathrm{l}$ of $5 \%$ glucose (wt/ vol.), according to the manufacturer's instruction. The mixture was intravenously injected into diabetic mice via the tail vein as we recently described [22].

Two months after induction of diabetes, mice were subjected to the following experiments. There were eight to ten mice in each group.

Echocardiography Mice were lightly anaesthetised with inhaled isoflurane $(1 \%)$. Echocardiographic analysis was performed using a $40 \mathrm{MHz}$ linear array transducer (MS-550D; VisualSonics, Toronto, ON, Canada) attached to a preclinical ultrasound system (Vevo 2100; Visual Sonics) with nominal in-plane spatial resolution of $40 \mu \mathrm{m}$ (axial) $\times 80 \mu \mathrm{m}$ (lateral) [23], as we recently described [24]. To assess diastolic function, we obtained an apical four-chamber view of the left ventricle. Pulsed wave Doppler measurements were obtained in the apical view with a cursor at mitral valve inflow: maximal 
early (E) and late (A) transmitral velocities in diastole. Coronary blood flow was assessed by Doppler measurement of the left anterior descending artery flow under a modified fourchamber view as previously described [25]. To measure maximal coronary flow, mice were anaesthetised with $3 \%$ isoflurane vaporised with $100 \%$ oxygen.

LV pressure and volume measurements Mice were anaesthetised with ketamine $(100 \mathrm{mg} / \mathrm{kg})$ and xylazine $(5 \mathrm{mg} / \mathrm{kg}$, i.p.) and ventilated. The chest was opened and a Scisense mouse PV catheter (FTE-1212B-4518, 1.2F; Scisense, London, ON, Canada) was directly inserted into the left ventricle via the apex to measure LV pressure and volume as we described recently $[24,26]$.

Histological analysis The collagen content and cardiomyocyte cross-sectional area were assessed as described in our recent report [26, 27].

Endothelial cells were identified using an antibody against CD31 (DAKO, Burlington, ON, Canada). In brief, tissue sections were incubated in $0.3 \%$ hydrogen peroxide for $20 \mathrm{~min}$ to block endogenous peroxidase activity. To prevent nonspecific binding, sections were pre-incubated for $30 \mathrm{~min}$ in PBS containing horse serum. The sections were then incubated with rabbit anti-human CD31 antibody (1:200) and subsequently incubated with swine anti-rabbit IgG antibody $(1: 100$; DAKO) followed by incubation with rabbit peroxidase antiperoxidase complex (1:50, DAKO). CD31 was visualised by staining with 3-diaminobenzidine substrate, which produces a yellow-brown colour. Sections were counterstained with haematoxylin.

Determination of oxidative stress in diabetic mouse hearts The formation of reactive oxygen species (ROS) in heart tissue lysates was measured by using 2,7dichlorodihydro-fluorescein diacetate (DCF-DA; Invitrogen, Carlsbad, CA, USA) as an indicator [28]. The protein oxidation in heart tissues was assessed by measuring the protein carbonyl content using a commercial assay kit (Cayman Chemical, Ann Arbor, MI, USA) following manufacturer's instructions.

Cardiomyocyte cultures Cardiomyocytes were isolated from adult rats and mice and cultured as described [28].

Transfection of cardiac microvascular endothelial cells Mouse cardiac microvascular endothelial cells were purchased from CELLutions Biosystems (Cedarlane Laboratories, Hornby, ON, Canada). A chemically modified antisense oligonucleotide (antagomir; GenePharm, Shanghai, China) was used to inhibit miR-195 expression and a scrambled oligonucleotide (GenePharm) was used as a control. Transfection was conducted by using TransMessenger transfection reagent (Qiagen, Toronto, ON, Canada) according to the manufacturer's instructions as described previously [21].

Adenoviral infection Cardiomyocytes and endothelial cells were infected with adenoviral vectors containing miR-195 (Ad-miR-195; Vigene Biosciences, Rockville, MD, USA) or $\beta$-gal (Ad-gal; Vector Biolabs, Philadelphia, PA, USA) as a control at a multiplicity of infection (MOI) of 50-100 PFU/ cell, as we previously described [28].

Two-dimensional cardiac microvascular endothelial cell culture Angiogenesis of cardiac endothelial cells was assessed in vitro using the Endothelial Tube Formation Assay (Cell Biolabs, San Diego, CA, USA), following the manufacturer's instructions. Four hours after seeding, the formation of capillary-like structures from cardiac endothelial cells was examined on Matrigel (Cell Biolabs, San Diego, CA, USA). The formation of the capillary-like network was assessed by counting the number of connections between three or more capillary-like structures and determining the number of capillary connections per field. The total length of tubes per field was quantified by image analysis with an image analysis system (SigmaPro, Fort Collins, CO, USA).

MiR-195 expression assay miR-195 expression assay was performed by using the miRNA plate assay kit (Signosis, Sunnyvale, CA, USA) according to the manufacturer's instruction, as described in our recent report [21].

Active caspase-3 As described previously [28], caspase-3 activity in heart tissues was measured using a caspase-3 fluorescence assay kit (Biomol Research Laboratories, Plymouth Meeting, PA, USA).

Measurement of cellular DNA fragmentation DNA fragmentation was measured using a Cellular DNA Fragmentation ELISA kit (Roche Applied Science, Indianapolis, IN, USA) according to the manufacturer's instructions.

Real-time RT-PCR Total RNA was extracted from heart tissues using Trizol Reagent (Gibco-BRL, Rockville, MD, USA) following the manufacturer's instructions. Real-time RT-PCR was performed to analyse mRNA expression for Anp (also known as Nppa), $\beta$-Mhc (also known as Myh7) collagen I and III, copGFP and Gapdh as described previously $[26,27]$.

Western blot analysis The protein levels of Sirt1, BCL-2 and GAPDH were determined by western blot analysis using specific antibodies (1:1,000 dilutions; Cell Signaling, Danvers, MA, USA) against the respective corresponding proteins. 
Statistical analysis All data were given as means \pm SD. ANOVA followed by the Newman-Keuls test was performed for multi-group comparisons. A value of $p<0.05$ was considered statistically significant.

\section{Results}

MiR-195 is upregulated in diabetic hearts MiR-195 was upregulated at 10 days and further increased in mouse hearts at 2 months after STZ injection (Fig. 1a). MiR-195 levels were also elevated in $d b / d b$ mouse hearts (electronic supplementary material [ESM] Fig. 1) and in cardiomyocytes isolated from $d b / d b$ mice (Fig. 1b). Diabetes increased miR-195 expression
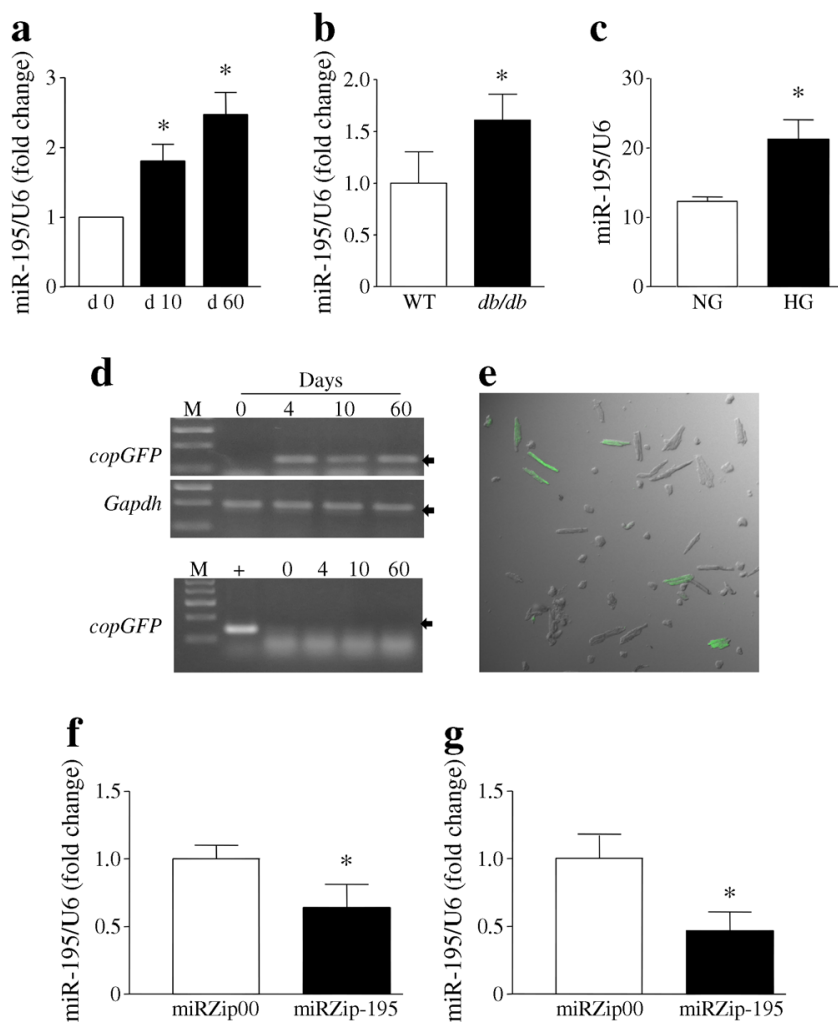

Fig. 1 MiR-195 expression and silencing in hearts and cardiomyocytes. (a) MiR-195 levels in mouse heart tissues at 0,10 and 60 days (d) after STZ injection. (b) MiR-195 levels in cardiomyocytes isolated from $d b / d b$ (male, 3 months old) and $d b / \pm$ mice as wild-type (WT) controls. (c) miR195 levels in adult rat cardiomyocytes incubated with normal (NG) or high glucose $(\mathrm{HG})$ for $24 \mathrm{~h}$. (d-g) Heart tissues were collected from diabetic mice at 4, 10 and 60 days after delivery of miRZip-195. (d) A representative RT-PCR showing copGFP mRNA is shown, with representative PCR to determine copGFP mRNA contamination, using total RNA without reverse transcription directly for PCR, below. M, DNA marker; +, positive control, miRZip-195. (e) Representative fluorescent confocal microscopy image showing copGFP fluorescent signals (green) in cardiomyocytes isolated from mice receiving miRZip-195 (4 days after delivery). (f, g) miR-195 expression levels in heart tissues at 10 (f) and 60 (g) days after miRZip-195 delivery. The miR-195 levels were normalised to U6 small nuclear RNA. Data are means $\pm \mathrm{SD}, n=8$ or 3 different cultures. ${ }^{*} p<0.05$ in liver and kidney of STZ-injected mice (ESM Fig. 2). Similarly, incubation with high glucose $(30 \mathrm{mmol} / \mathrm{l})$ for $24 \mathrm{~h}$ significantly increased the levels of miR-195 in rat cardiomyocytes (Fig. 1c).

In vivo silencing of miR-195 in diabetic hearts To achieve long-lasting silencing of miR-195 in vivo, we delivered miRZip-195 systemically to STZ-induced diabetic mice, with a second dose administered to the same mice 10 days after the first injection. Since miRZip-195 also expresses copGFP, we measured copGFP expression in heart tissues to determine the efficiency of delivery. copGFP mRNA was detected at 4 days, 10 days and 2 months after the first injection in total RNA isolated from the heart; however, no copGFP DNA was observed in the same total RNA (Fig. 1d). This result indicates expression of miRZip construct in mouse hearts in vivo. To verify the expression of copGFP protein, we identified copGFP signals in at least $10 \%$ of cardiomyocytes isolated from heart tissues of mice receiving miRZip-195 using confocal fluorescence microscopy (Fig. 1e).

To assess the silencing efficiency of miRZip-195, we measured miR-195 levels in mouse hearts 10 days and 2 months after the first injection. The miRZip-195 significantly reduced the levels of miR-195 (Fig. 1f, g) but not miR-15a (another member in the same family) (ESM Fig. 3), compared with miRZip00 construct. These results suggest that two injections of miRZip-195 are sufficient to achieve a specific and longlasting silencing effect in diabetic hearts. Injections of miRZip-195 also reduced the levels of miR-195 in liver and kidney of STZ-injected mice (ESM Fig. 2).

Silencing of miR-195 increased the insulin sensitivity (ESM Fig. 4), reduced the levels of TNF- $\alpha$ protein in plasma (ESM Fig. 5) and inhibited Tnf- $\alpha$ (also known as Tnf) and Il$1 \beta$ (Illb) mRNA expression in skeletal muscles of diabetic mice (ESM Fig. 5).

miRZip00 delivered via nanoparticles did not affect liver (as measured by aspartate transaminase [AST] and alanine transaminase [ALT] activity) or renal function (creatinine levels) in diabetic mice (ESM Fig. 6), suggesting no toxic effects on liver and kidney. The miRZip-195 prevented liver injury in diabetic mice as determined by a reduction in ALT and AST activity.

Silencing miR-195 did not affect blood glucose, blood pressure or body weight in either sham or STZ-injected mice (ESM Table 1). There were no other obvious changes, including activity and dietary ingestion, in mice receiving either miRZip-195 or miRZip00.

Downregulation of miR-195 increases its target expression in diabetic hearts Sirt1 and BCL-2 protein levels were significantly reduced in STZ-induced and $d b / d b$ diabetic compared with non-diabetic mouse hearts (Fig. 2a, b, e-g). Silencing miR-195 increased the levels of Sirt1 and BCL-2 in the 
a

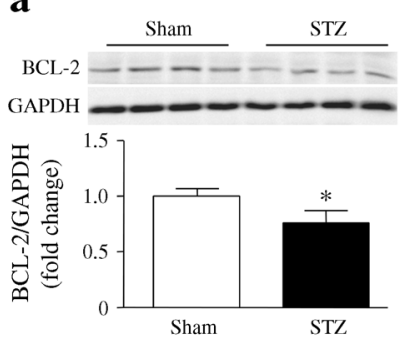

c
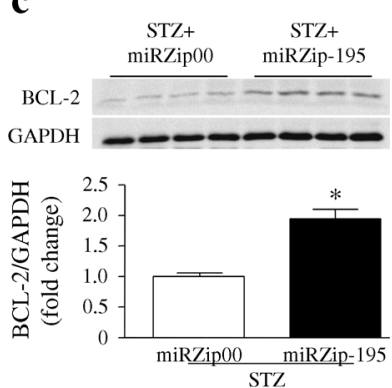

b

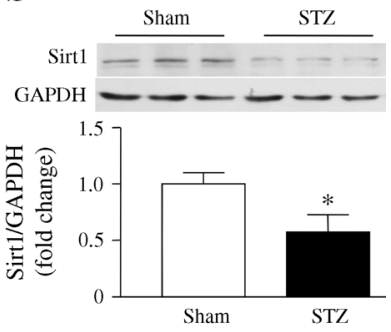

d
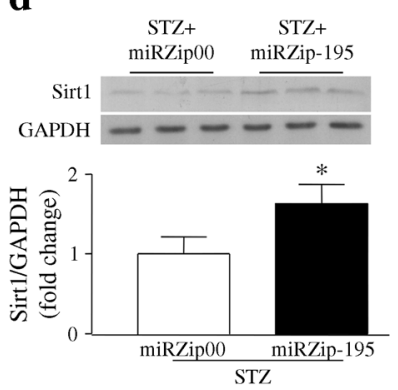

e

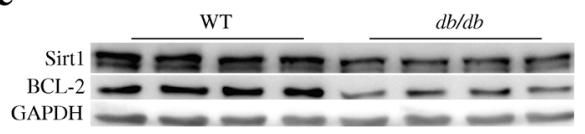

f
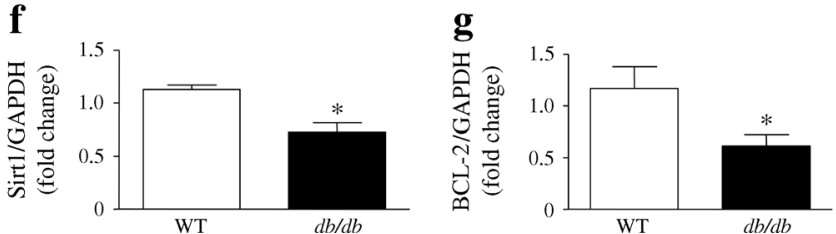

Fig. 2 The protein levels of BCL-2 and Sirt1 in diabetic mouse hearts. (a-d) Western blot analysis was performed to determine the protein levels of BCL-2 (a, c) and Sirt1 $(\mathbf{b}, \mathbf{d})$ in hearts after 60 days of STZ injection. Representative western blots are shown from four (BCL-2) or three (Sirt1) out of eight different hearts in each group. Quantification of BCL-2 and Sirt1 protein levels normalised to GAPDH is shown. (e) Representative western blots for BCL-2 and Sirt1 protein from four different $d b / d b$ and wild-type (WT) mouse hearts. (f, g) Quantification of Sirt1 and BCL-2 protein levels normalised to GAPDH. Data are means \pm $\mathrm{SD}, n=6-8 .{ }^{*} p<0.05$

diabetic hearts (Fig. 2c, d), demonstrating that miR-195 represses BCL-2 and Sirt1 protein expression. Silencing of miR-195 also prevented the downregulation of Sirt1 and BCL-2 protein in the liver of STZ-treated mice (ESM Fig. 7).

\section{Knockdown of miR-195 attenuates myocardial dysfunc-} tion in diabetic mice Myocardial function was assessed by measuring LV pressure and volume in mice 2 months after the first injection of miRZip constructs (ESM Table 2). Although there was no change in maximal positive first derivatives of $\mathrm{LV}$ pressure $\left(+\mathrm{d} P / \mathrm{d} t_{\max }\right.$, ESM Table 2$)$, maximal negative first derivatives of $\mathrm{LV}$ pressure $\left(-\mathrm{d} P / \mathrm{d} t_{\min }\right)$ were significantly decreased in diabetic hearts, consistent with the diastolic dysfunction usually seen in early stage of diabetes [3]. However, the change in $-\mathrm{d} P / \mathrm{d} t_{\min }$ was attenuated in the hearts of diabetic who received miRZip-195 (Fig. 3a). Delivery of miRZip195 did not influence myocardial function in sham mice.

Echocardiographic analysis revealed a decrease in the systolic function index per cent fractional shortening in diabetic mouse hearts compared with the sham mouse hearts (Fig. 3b and ESM Table 3). Inhibition of miRZip-195 restored the systolic function in diabetic mice. Tissue Doppler analysis showed that the E/A ratio of about 2 in sham mice was consistent with other investigator's result from wild-type adult mice [29]. In line with haemodynamic analysis, the E/A ratio was significantly decreased in diabetic mice, indicative of diastolic dysfunction (Fig. 3c). Delivery of miRZip-195 increased the E/A ratio in diabetic mice but not in sham mice. Thus, inhibition of miR-195 reduces myocardial dysfunction in diabetic mice.

Silencing miR-195 reduces cardiac hypertrophy, oxidative damage and caspase- 3 activity in diabetic mice Two months after STZ injection, the ratio of heart weight to body weight was increased in diabetic mice compared with sham mice, suggesting hypertrophy in diabetic hearts (ESM Table 1). Histological analysis of cardiomyocyte cross-sectional areas

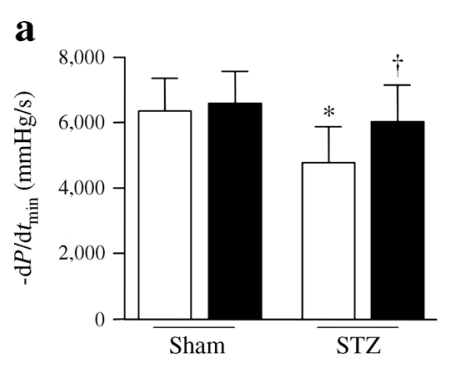

b
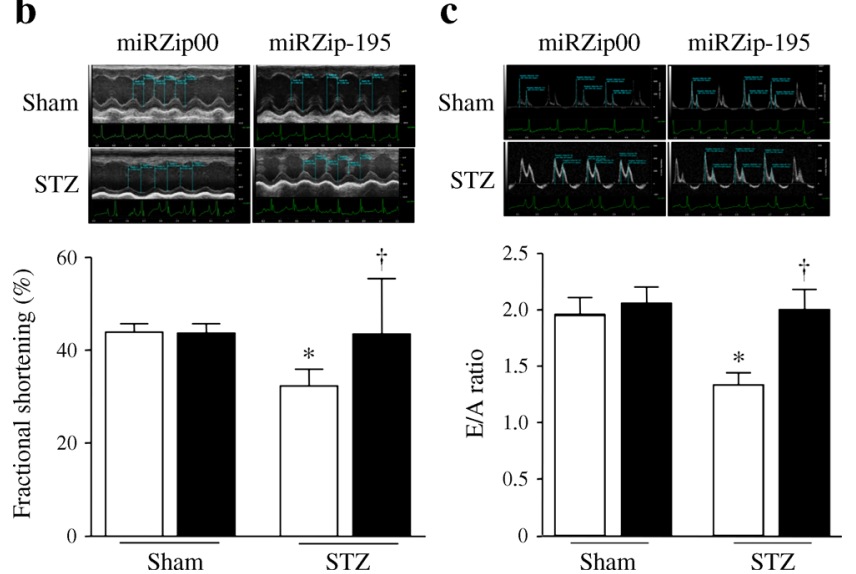

Fig. 3 Myocardial dysfunction in diabetic mice. Cardiac haemodynamic measurements were conducted 2 months after STZ injection. (a) Maximal negative first derivatives of LV pressure $\left(-\mathrm{d} P / \mathrm{d} t_{\min }\right) .(\mathbf{b}, \mathbf{c})$ Echocardiography was conducted. (b) Representative picture for M-mode with quantification of the fractional shortening. (c) Representative pulsed wave Doppler curves and the E/A ratio. White bars, miRZip00; black bars, miRZip-195. Data are means \pm SD,$n=8 . * p<0.05$ vs Sham + miRZip00; ${ }^{\dagger} p<0.05$ vs STZ+miRZip00 
confirmed that cardiomyocyte size was increased in diabetic vs non-diabetic hearts (Fig. 4a and ESM Table 1). These effects of diabetes on cardiac hypertrophy were inhibited by silencing miR-195. Similarly, miRZip-195 decreased the mRNA levels of hypertrophic genes (Anp and $\beta-M h c)$ in diabetic hearts (Fig. 4b, c).

Silencing miR-195 did not significantly affect myocardial collagen deposition (data not shown). As miR-195 is known to target important extracellular matrix components and regulatory genes, including those for collagens, proteoglycans, elastin and proteins associated with elastic microfibrils [30], we analysed the expression of these genes in mouse hearts. Diabetes increased the levels of biglycan, Timp1, Mmp2 and $T g f-\beta 1$ mRNA in the heart; the raised levels were significantly decreased by miRZip-195 (Fig. 4d-g). In contrast, the levels of elastin and Mmp9 mRNA were not changed in diabetic hearts (data not shown).

We examined ROS production, oxidative damage and caspase-3 activity as indicators of apoptosis. Silencing miR195 reduced ROS production (Fig. 5a) and decreased protein carbonyl content in diabetic mouse hearts (Fig. 5b), suggesting that inhibition of miR-195 attenuates oxidative damage.
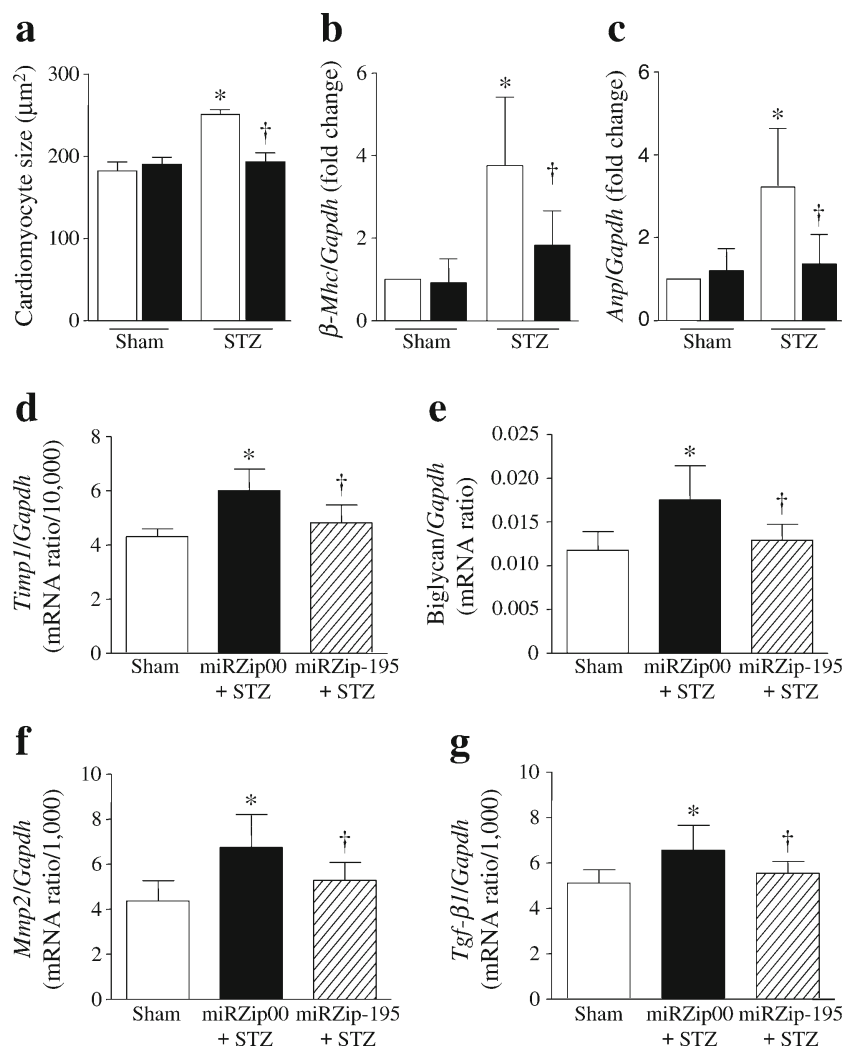

Fig. 4 Hypertrophic changes and extracellular matrix components and regulatory genes in diabetic mouse hearts. (a) Quantification of cardiomyocyte cross-sectional areas. (b, c) The levels of $\beta$-Mhc (b) and Anp (c) mRNA were measured in heart tissues and normalised to Gapdh. White bars, miRZip00 and black bars, miRZip-195. (d-g) Expression of Timp1 (d), biglycan (e), Mmp2 (f) and $T g f-\beta 1$ (g) mRNA. Data are means \pm SD, $n=8 .{ }^{*} p<0.05$ vs Sham + miRZip00; ${ }^{\dagger} p<0.05$ vs STZ + miRZip00
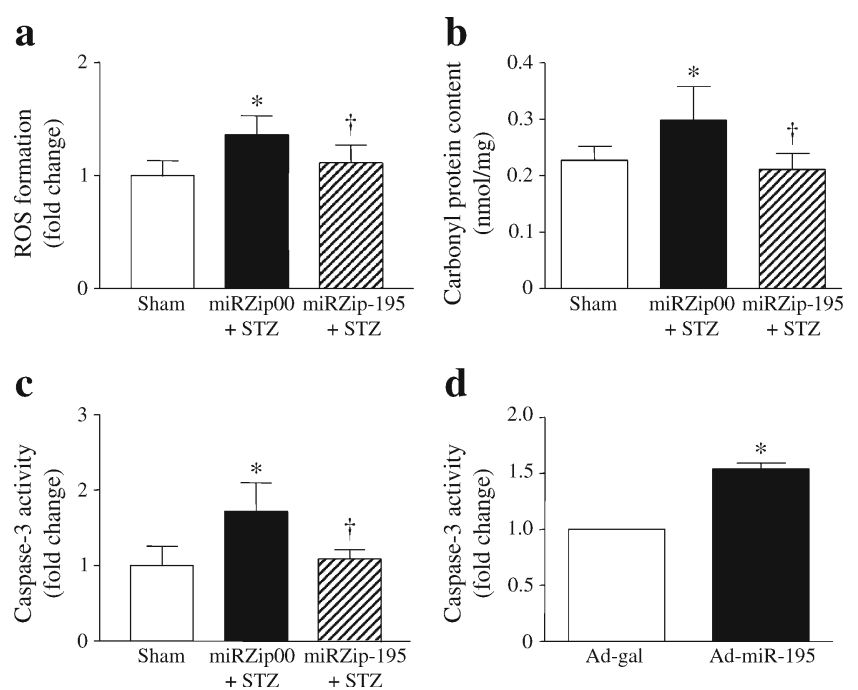

Fig. 5 Assessment of oxidative stress and apoptosis in diabetic mouse hearts and cardiomyocytes. (a) ROS formation was measured using DCFDA in heart tissue lysates. (b) Protein oxidation was determined by measuring carbonyl protein content. (c) Apoptosis was determined by caspase- 3 activation in hearts. Data are means $\pm \mathrm{SD}, n=6 .{ }^{*} p<0.05$ vs Sham; ${ }^{\dagger} p<0.05$ vs STZ+miRZip00. (d) Mouse cardiomyocytes were isolated and infected with Ad-miR-195 or Ad-gal as a control. Twenty-four hours later, caspase- 3 activity was measured. Data are means \pm SD from three different experiments. ${ }^{*} p<0.05$

Knockdown of miR-195 also inhibited apoptosis in diabetic hearts (Fig. 5c). In vitro study confirmed that upregulation of miR-195 sufficiently induced apoptosis in cultured cardiomyocytes (Fig. 5d).

Inhibition of miR-195 improves coronary blood flow and increases myocardial capillary density in diabetic mice Consistently, we showed that the maximal coronary blood flow was decreased in diabetic hearts and was associated with a reduction in capillary density. Silencing miR-195 significantly improved the maximal coronary blood flow and increased myocardial capillary density in diabetic mice (Fig. 6a-d), suggesting that upregulation of miR-195 contributes to the impairment of coronary circulation in diabetes.

Upregulation of miR-195 decreases the in vitro tube formation of cardiac endothelial cells Cardiac microvascular endothelial cells were infected with Ad-miR-195 or with Ad-gal as a control. After infection, the cells were seeded onto Matrigel-coated 96-well plates. The tube formation reached an optimal level after $4 \mathrm{~h}$ of culture. Compared with Ad-gal, the tube formation was decreased in Ad-miR-195-infected cardiac endothelial cells (Fig. 7a). Quantitative analysis showed that the total length of tube between connections and the number of capillary connections were significantly decreased in AdmiR-195-infected cardiac endothelial cells compared with Ad-gal infected cells (Fig. 7b, c). Thus, upregulation of miR-195 impairs angiogenesis of cardiac endothelial cells. 


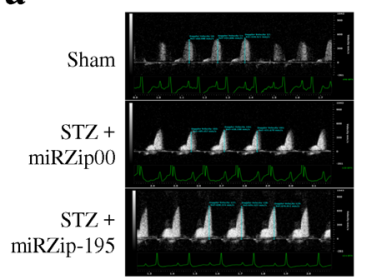

b

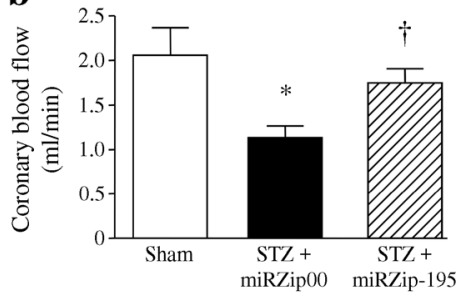

c

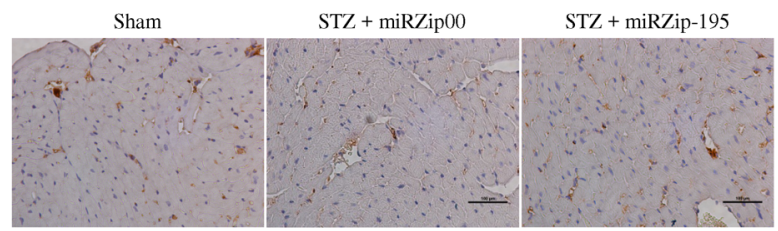

d

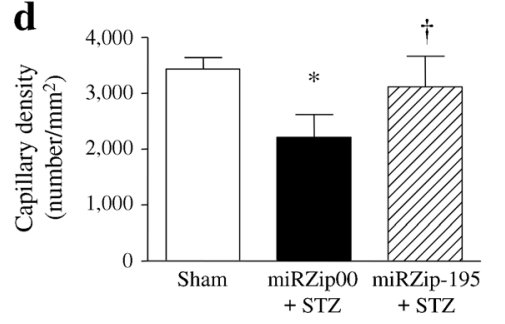

Fig. 6 Coronary blood flow and myocardial capillary density in diabetic mice. Two months after STZ injection, maximal coronary blood flow was examined in diabetic mice. (a) Representative pulse wave Doppler coronary flow curves. (b) Quantification of the coronary blood flow in diabetic mice. (c) Representative micrographs showing CD31 staining (yellow-brown) in myocardial endothelial cells. (d) Quantification of myocardial capillary density. Data are means $\pm \mathrm{SD}, n=6$. ${ }^{*} p<0.05$ vs Sham and ${ }^{\dagger} p<0.05$ vs STZ+miRZip00

Inhibition of miR-195 prevents apoptosis in endothelial cells during palmitate stimulation To examine whether miR-195 plays a direct role in endothelial cell injury in diabetes, we transfected cardiac microvascular endothelial cells with miR-195 antagomir or a scrambled oligo as a control, and then incubated them with palmitate (NEFA, $100 \mu \mathrm{mol} / \mathrm{l}$ ) or oleate as a control for $24 \mathrm{~h}$. As shown in Fig. 7d, e, palmitate induced apoptosis in endothelial cells. Transfection with miR-195 antagomir prevented palmitate-induced apoptosis. This result provides further evidence to support the role of miR-195 in diabetes-induced endothelial cell injury.

\section{Discussion}

The major findings of the present study are that therapeutic silencing of miR-195 reduces myocardial hypertrophy, improves coronary circulation and attenuates myocardial dysfunction in STZ-induced diabetic mice. These effects are associated with decreased oxidative stress and apoptosis and increased endothelial cell angiogenesis in diabetic hearts. Thus, this study demonstrates an important role for miR-195 in diabetic cardiomyopathy and highlights the therapeutic a
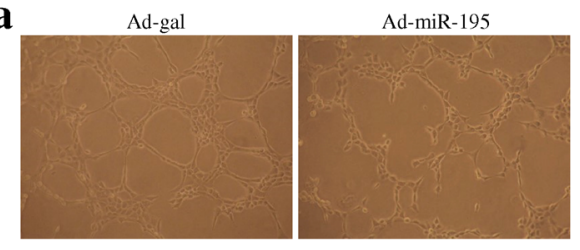

b
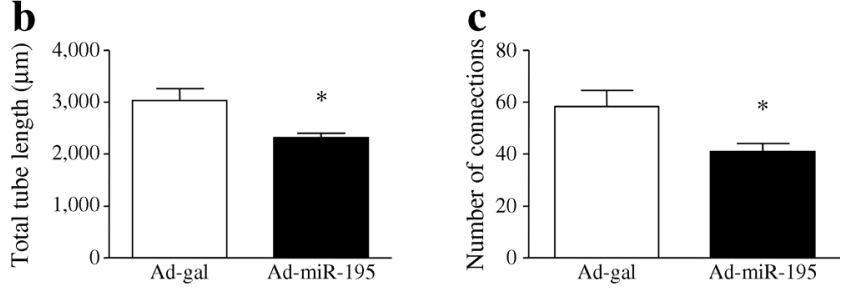

d
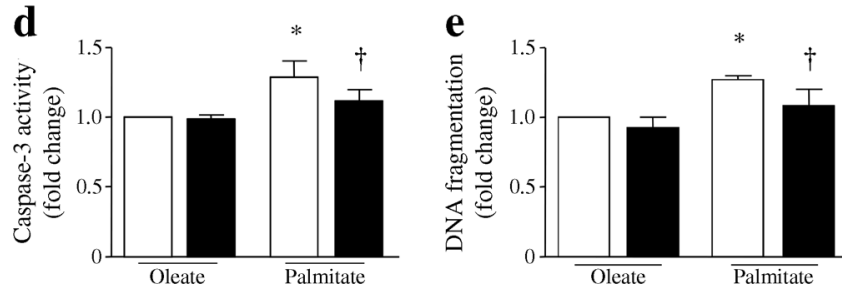

Fig. 7 Assessment of in vitro angiogenesis and apoptosis in mouse endothelial cells. (a-c) Cardiac microvascular endothelial cells were infected with Ad-miR-195 or Ad-gal as a control, and seeded onto Matrigelcoated 96-well plates for $4 \mathrm{~h}$. (a) Representative micrographs of in vitro angiogenesis of endothelial cells. (b) The total length of tube between connections. (c) The number of capillary connections. Data are means \pm SD from three independent experiments. ${ }^{*} p<0.05$ vs Ad-gal. (d, e) Cardiac microvascular endothelial cells were transfected with miR-195 antagomir or a scrambled oligo and then incubated with palmitate or oleate $(100 \mu \mathrm{mol} / \mathrm{l})$ for $24 \mathrm{~h}$. Caspase-3 activity was determined (d) and DNA fragmentation was assessed (e). White bars, scrambled oligos; black bars, antagomir. Data are means \pm SD from four independent experiments. ${ }^{*} p<0.05$ vs oleate + scrambled oligos; ${ }^{\dagger} p<0.05$ vs palmitate + scrambled oligos

potential of miR-195 silencing to reduce diabetic cardiomyopathy.

Diabetes has been reported to regulate miR-195 expression in animal models. A recent study showed a reduction in miR195 in the kidney of STZ-induced type 1 diabetic mice [31]. However, upregulation of miR-195 was revealed in liver in a rat model of spontaneous type 2 diabetes [32] and in retinas of STZ-induced diabetic rats [33]. These studies suggest that there is a tissue-dependent differential expression of miR195 in diabetes. In the present study, we demonstrate that both type 1 and type 2 diabetes promote expression of miR-195 in the heart. This is supported by our in vitro findings that incubation with high glucose upregulates miR-195 expression in cardiomyocytes, together with our recent report which demonstrated that high NEFA directly induced miR-195 expression in cardiomyocytes [21]. MiR-195 is also upregulated in liver and kidney of STZ-induced mice.

Increased levels of miR-195 have been implicated in apoptosis and hypertrophy in cardiomyocytes under stress [21, 34]. In this study, upregulation of miR-195 in diabetic hearts 
was associated with oxidative stress, apoptosis, myocardial hypertrophy and dysfunction as well as a reduction in coronary blood flow. Importantly, silencing of miR-195 reduces oxidative damage, apoptosis and hypertrophy and restores coronary blood flow in diabetic hearts, leading to the improvement of myocardial function. Thus, the present study demonstrates that miR-195 makes a significant contribution to diabetic cardiomyopathy. Inhibition of miR-195 has also been demonstrated to attenuate renal injury in STZ-induced mice [31] and may be involved in retinopathy in STZ-induced rats [33]. We show that silencing of miR-195 reduces liver injury and systemic inflammation in diabetic mice as well. Thus, miR-195 may represent a potential therapeutic target for diabetic complications.

Although the underlying mechanisms by which silencing miR-195 provides cardiac protection in diabetes remain to be determined, it seems that multiple mechanisms might be involved. First, miR-195 targets and represses expression of BCL-2 and Sirt1 in cardiomyocytes [21, 34]. Previous studies have revealed that levels of these proteins are reduced in diabetic hearts $[35,36]$ and in the present study we confirm this downregulation. Importantly, silencing of miR-195 increases the levels of BCL-2 and Sirt1, providing further evidence to support the view that miR-195 targets and inhibits BCL-2 and Sirt1 expression in a mouse model of type 1 diabetes. BCL-2 is a well-known anti-apoptotic protein [37]. Our recent study has demonstrated that knockdown of miR-195 increases levels of BCL-2 protein, thereby preventing apoptosis in cultured cardiomyocytes in response to high NEFA levels, an important feature of diabetes [21]. Thus, the protective effects of miRZip-195 in diabetic hearts may be related to upregulation of BCL-2. Sirt1, a class III histone/protein deacetylase, is thought to be an important factor in regulating cardiomyocyte survival $[21,38,39]$. The Sirt1 activator resveratrol improves cardiac function in animal models of diabetes [35, 40, 41]. Second, oxidative damage induced by ROS is critical in diabetic cardiomyopathy [3, 42]. The present study shows that silencing of miR-195 reduces oxidative stress in diabetic hearts and the reduction in oxidative damage may attenuate diabetic cardiomyopathy. In addition, miR-195 modulation of systemic inflammation, insulin sensitivity and injury to other organs, such as the liver, may also contribute to diabetic cardiomyopathy.

Our data suggest that protection of cardiac endothelial cells may represent another important mechanism by which inhibition of miR-195 reduces diabetic cardiomyopathy. Silencing miR-195 increases myocardial capillary density and restores maximal coronary blood flow in diabetic mice, suggesting that miR-195 may play a role in impairment of endothelial cell angiogenesis in the heart. Our experimental evidence showing that upregulation of miR-195 decreases tube formation in cardiac endothelial cells in vitro provides support for this hypothesis. Furthermore, knockdown of miR-195 prevents apoptosis in cultured cardiac endothelial cells. Thus, the protection of endothelial cell angiogenesis and prevention of endothelial cell death conferred by inhibition of miR-195 may contribute to the increase in myocardial capillary density and subsequently improve coronary blood flow in diabetic hearts, leading to attenuation of diabetic cardiomyopathy.

Our study shows that administration of miRZip-195 knocked down miR-195 but not miR-15a, another member of the miR-15 family. This finding may at least to some extent exclude the target-off effects of miRZip-195 on other miRs. We used a nanoparticle-based transfection reagent to systemically deliver miRZip-195 to mice. Although the exact components of this reagent are unknown due to commercial secrecy, we did not observe any obvious health problems, or liver and kidney toxicity in animals receiving it, suggesting that it had no significant side-effects. Nevertheless, there is no information as to where the nanoparticles ended up in the mice or how much of the nanoparticles were actually delivered to the heart. Our data suggest that at least $10 \%$ of cardiomyocytes in the heart expressed copGFP after systemic delivery. Thus, in the future it is necessary to study whether other transfection reagents provide better efficiency of delivery.

It is important to mention that other miRs may also be involved in the development of diabetic cardiomyopathy, such as miR-133 [43], miR-1 [44] and miR-499 [45]. It is currently unknown whether simultaneously silencing multiple miRs provides better protective effects in the diabetic heart. This requires further study for clarification.

In summary, we showed that in both type 1 and type 2 diabetes, miR-195 expression was induced and BCL-2 and Sirt1 expression was decreased in the heart. Therapeutic silencing of miR-195 reduced myocardial hypertrophy and apoptosis, increased myocardial capillary density and improved coronary blood flow and myocardial function in a mouse model of STZ-induced type 1 diabetes. Thus, silencing miR195 may be a new therapy with which to reduce diabetic cardiomyopathy.

Funding This study was supported by grants from the Canadian Institutes of Health Research (MOP-133657) and the National Natural Science Foundation of China ( 81170204 and 81470499$)$ and also partly by the Western Department of Medicine Program of Experimental Medicine (POEM) Research Award. The research in GCF's laboratory is supported by NIH R01 grant (grant number HL-087861). TP is a recipient of a New Investigator Award from the Canadian Institutes of Health Research.

Duality of interest The authors declare that there is no duality of interest associated with this manuscript.

Contribution statement DZ, JM, YY, ML, RN, GW and JL performed experiments, contributed to data acquisition and drafted the manuscript. DZ and TP designed this study. RC, GCF, JCL and TP analysed data and revised the manuscript. All authors approved the final version of the manuscript. TP is the guarantor of this work. 


\section{References}

1. International Diabetes Federation (2009) IDF diabetes atlas. International Diabetes Federation, Brussels

2. Mazzone T, Chait A, Plutzky J (2008) Cardiovascular disease risk in type 2 diabetes mellitus: insights from mechanistic studies. Lancet 371:1800-1809

3. Boudina S, Abel ED (2007) Diabetic cardiomyopathy revisited. Circulation 115:3213-3223

4. Meister G, Tuschl T (2004) Mechanisms of gene silencing by double-stranded RNA. Nature 431:343-349

5. Brennecke J, Hipfner DR, Stark A, Russell RB, Cohen SM (2003) Bantam encodes a developmentally regulated microRNA that controls cell proliferation and regulates the proapoptotic gene hid in Drosophila. Cell 113:25-36

6. Xu P, Vernooy SY, Guo M, Hay BA (2003) The Drosophila microRNA Mir-14 suppresses cell death and is required for normal fat metabolism. Curr Biol 13:790-795

7. Ambros V (2004) The functions of animal microRNAs. Nature 431:350-355

8. Cimmino A, Calin GA, Fabbri M et al (2005) miR-15 and miR-16 induce apoptosis by targeting BCL2. Proc Natl Acad Sci U S A 102:13944-13949

9. Gupta A, Gartner JJ, Sethupathy P, Hatzigeorgiou AG, Fraser NW (2006) Anti-apoptotic function of a microRNA encoded by the HSV-1 latency-associated transcript. Nature 442:82-85

10. Fiedler J, Jazbutyte V, Kirchmaier BC et al (2011) MicroRNA-24 regulates vascularity after myocardial infarction. Circulation 124:720-730

11. Lagendijk AK, Goumans MJ, Burkhard SB, Bakkers J (2011) MicroRNA-23 restricts cardiac valve formation by inhibiting Has2 and extracellular hyaluronic acid production. Circ Res 109:649-657

12. Porrello ER, Johnson BA, Aurora AB et al (2011) MiR-15 family regulates postnatal mitotic arrest of cardiomyocytes. Circ Res 109:670-679

13. Boon RA, Iekushi K, Lechner S et al (2013) MicroRNA-34a regulates cardiac ageing and function. Nature 495:107-110

14. Chen J, Huang ZP, Seok HY et al (2013) mir-17-92 cluster is required for and sufficient to induce cardiomyocyte proliferation in postnatal and adult hearts. Circ Res 112:1557-1566

15. Huang ZP, Chen J, Seok HY et al (2013) MicroRNA-22 regulates cardiac hypertrophy and remodeling in response to stress. Circ Res 112:1234-1243

16. Li RC, Tao J, Guo YB et al (2013) In vivo suppression of microRNA-24 prevents the transition toward decompensated hypertrophy in aortic-constricted mice. Circ Res 112:601-605

17. Mishra PK, Tyagi N, Kumar M, Tyagi SC (2009) MicroRNAs as a therapeutic target for cardiovascular diseases. J Cell Mol Med 13: 778-789

18. Quiat D, Olson EN (2013) MicroRNAs in cardiovascular disease: from pathogenesis to prevention and treatment. J Clin Invest 123:11-18

19. van Rooij E, Sutherland LB, Liu N et al (2006) A signature pattern of stress-responsive microRNAs that can evoke cardiac hypertrophy and heart failure. Proc Natl Acad Sci U S A $103: 18255-18260$

20. Chen H, Untiveros GM, McKee LA et al (2012) Micro-RNA-195 and -451 regulate the LKB1/AMPK signaling axis by targeting MO25. PLoS One 7:e41574

21. Zhu H, Yang Y, Wang Y, Li J, Schiller PW, Peng T (2011) MicroRNA-195 promotes palmitate-induced apoptosis in cardiomyocytes by down-regulating Sirt1. Cardiovasc Res 92:75-84
22. Zhang Y, Peng T, Zhu H et al (2010) Prevention of hyperglycemiainduced myocardial apoptosis by gene silencing of Toll-like receptor-4. J Transl Med 8:133

23. Foster FS, Mehi J, Lukacs M et al (2009) A new 15-50 MHz arraybased micro-ultrasound scanner for preclinical imaging. Ultrasound Med Biol 35:1700-1708

24. Ma J, Wei M, Wang Q et al (2012) Deficiency of Capn4 gene inhibits nuclear factor-kappaB (NF-kappaB) protein signaling/ inflammation and reduces remodeling after myocardial infarction. J Biol Chem 287:27480-27489

25. Katz PS, Trask AJ, Souza-Smith FM et al (2011) Coronary arterioles in type 2 diabetic $(\mathrm{db} / \mathrm{db})$ mice undergo a distinct pattern of remodeling associated with decreased vessel stiffness. Basic Res Cardiol 106:1123-1134

26. Li Y, Ma J, Zhu H et al (2011) Targeted inhibition of calpain reduces myocardial hypertrophy and fibrosis in mouse models of type 1 diabetes. Diabetes 60:2985-2994

27. Li J, Zhu H, Shen E, Wan L, Arnold JM, Peng T (2010) Deficiency of rac1 blocks NADPH oxidase activation, inhibits endoplasmic reticulum stress, and reduces myocardial remodeling in a mouse model of type 1 diabetes. Diabetes 59:2033-2042

28. Shen E, Li Y, Shan L et al (2009) Rac1 is required for cardiomyocyte apoptosis during hyperglycemia. Diabetes 58:2386-2395

29. Zhou YQ, Foster FS, Parkes R, Adamson SL (2003) Developmental changes in left and right ventricular diastolic filling patterns in mice. Am J Physiol Heart Circ Physiol 285:H1563-H1575

30. Zampetaki A, Attia R, Mayr U et al (2014) Role of miR-195 in aortic aneurysmal disease. Circ Res 115:857-866

31. Chen YQ, Wang XX, Yao XM et al (2012) Abated microRNA-195 expression protected mesangial cells from apoptosis in early diabetic renal injury in mice. J Nephrol 25:566-576

32. Herrera BM, Lockstone HE, Taylor JM et al (2010) Global microRNA expression profiles in insulin target tissues in a spontaneous rat model of type 2 diabetes. Diabetologia 53:1099-1109

33. Mortuza R, Feng B, Chakrabarti S (2014) miR-195 regulates SIRT1-mediated changes in diabetic retinopathy. Diabetologia 57:1037-1046

34. Liu L, Chen L, Xu Y, Li R, Du X (2010) microRNA-195 promotes apoptosis and suppresses tumorigenicity of human colorectal cancer cells. Biochem Biophys Res Commun 400:236-240

35. Sulaiman M, Matta MJ, Sunderesan NR, Gupta MP, Periasamy M, Gupta M (2010) Resveratrol, an activator of SIRT1, upregulates sarcoplasmic calcium ATPase and improves cardiac function in diabetic cardiomyopathy. Am J Physiol Heart Circ Physiol 298: $\mathrm{H} 833-\mathrm{H} 843$

36. Katare R, Caporali A, Zentilin L et al (2011) Intravenous gene therapy with PIM-1 via a cardiotropic viral vector halts the progression of diabetic cardiomyopathy through promotion of prosurvival signaling. Circ Res 108:1238-1251

37. Zhou F, Yang Y, Xing D (2011) Bcl-2 and Bcl-xL play important roles in the crosstalk between autophagy and apoptosis. FEBS J 278:403-413

38. Rane S, He M, Sayed D et al (2009) Downregulation of miR-199a derepresses hypoxia-inducible factor-1alpha and Sirtuin 1 and recapitulates hypoxia preconditioning in cardiac myocytes. Circ Res 104:879-886

39. Hsu CP, Zhai P, Yamamoto T et al (2010) Silent information regulator 1 protects the heart from ischemia/reperfusion. Circulation 122:2170-2182

40. Thirunavukkarasu M, Penumathsa SV, Koneru S et al (2007) Resveratrol alleviates cardiac dysfunction in streptozotocininduced diabetes: role of nitric oxide, thioredoxin, and heme oxygenase. Free Radic Biol Med 43:720-729

41. Zhang H, Morgan B, Potter BJ et al (2010) Resveratrol improves left ventricular diastolic relaxation in type 2 diabetes by inhibiting 
oxidative/nitrative stress: in vivo demonstration with magnetic resonance imaging. Am J Physiol Heart Circ Physiol 299:H985-H994

42. Cai L, Kang YJ (2001) Oxidative stress and diabetic cardiomyopathy: a brief review. Cardiovasc Toxicol 1:181-193

43. Chavali V, Tyagi SC, Mishra PK (2012) MicroRNA-133a regulates DNA methylation in diabetic cardiomyocytes. Biochem Biophys Res Commun 425:668-672
44. Yildirim SS, Akman D, Catalucci D, Turan B (2013) Relationship between downregulation of miRNAs and increase of oxidative stress in the development of diabetic cardiac dysfunction: junctin as a target protein of miR-1. Cell Biochem Biophys 67:1397-1408

45. Wang N, Yang C, Xie F et al (2012) Gadd45alpha: a novel diabetesassociated gene potentially linking diabetic cardiomyopathy and baroreflex dysfunction. PLoS One 7:e49077 